\title{
Grab Simulation Based on FSM for Astronaut Virtual Training
}

\author{
Hong $\mathrm{Hu}^{1}$, a , Jian Gang Chao ${ }^{1}$ and Jian Gang Liu ${ }^{2, b^{*}}$ \\ ${ }^{1}$ No.26 Beiqing Road, Haidian District, Beijing, China, China Astronaut Research and Training \\ Center \\ ${ }^{2}$ No.26 Beiqing Road, Haidian District, Beijing, China, Beijing Aerospace Control Center \\ ahhh20082976@126.com, b303904887@qq.com
}

\begin{abstract}
Keywords: Grab Simulation; FSM, Astronaut virtual Training
Abstract. Virtual hand grabbing operation simulation is one of the hardest interactive simulations in astronaut virtual training. This paper proposed a couple of non-contact grab determining rules and a grab simulation method based on finite state machine (FSM) to simulate the grabbing operation of virtual hand. The experiment result show that the proposed method has a high success rate and efficiency for grabbing operation, and it can be used for grabbing operation simulation in astronaut virtual training.
\end{abstract}

\section{Introduction}

With the development of virtual reality technology, a new training method based on virtual reality started to be applied in astronaut training [1,2]. Many astronaut virtual training systems were established using virtual hands as a main interactive tool $[3,4,5]$. The grabbing operation of virtual hand which is one of the most important interactive in astronaut virtual training is too complex to be simulated. On the one hand, grab determining rules suited for astronaut virtual environment should be formulated to determine whether the object is grabbed by a virtual hand. On the other hand, a typical grabbing operation has a lot of interactive states in virtual environment which would lead to different interactive results.

According to the special virtual weightless environment of astronaut virtual training, this paper proposed a couple of non-contact grab determining rules and a grab simulation method based on finite state machine (FSM). It divided the process of virtual hand grabbing operation into different states for simulation, and used the proposed rules to determine grab. The experiment showed that grabbing operation following our method has a high success rate and efficiency and can be used for grabbing operation simulation in astronaut virtual training.

\section{Grab Determining Rules}

$\mathrm{Grab}$ is an important part of the virtual hand interaction. In astronaut virtual training, virtual hand and the grabbed objects are all in virtual environment. Compared with grab in the real world, grab is more difficult in virtual environment because of the lack of force feedback. Grab determining rules are a kind of rules that used to determine whether a virtual hand grabs the object in virtual training world. On the one hand, grab determining rules cannot be too complicated, which could cause serious lag in render. On the other hand, they must meet the characteristic of astronaut virtual training.

The background of astronaut virtual training is set in the weightless environment of space, so all of the grabbed objects are weightless. A slight touch from virtual hand would lead to the movement of grabbed objects, which will result in failure to grab. Traditional grab determining rules based on collision detection are not suitable for weightless virtual environment. So, this paper developed two non-contact grab determining rules.

Rule One: the distance between thumb tip and one of the other fingertips must be less than a certain critical value, which is called the object grab trigger area.

Rule Two: the distance between the grabbed object and the virtual hand must be less than a certain critical value. 
It is determined that grab is successful only if the virtual hand and grabbed object meet the condition of Rule One and Rule Two at the same time. After successful grab, the grabbed object is attached to the virtual hand and moves with it together. The object will be released when the virtual hand does not satisfy Rule Two.

\section{Grab States Simulation Based on FSM}

Finite state machine (FSM) [6,7] is a good tool for states control of the virtual hand grab. In order to avoid the occurrence of interactive errors, a FSM was established to control the grab state transitions. The interactive model of FSM for virtual hand grab objects is shown in Fig. 1, where S represents the state, c represents the states transition conditions.

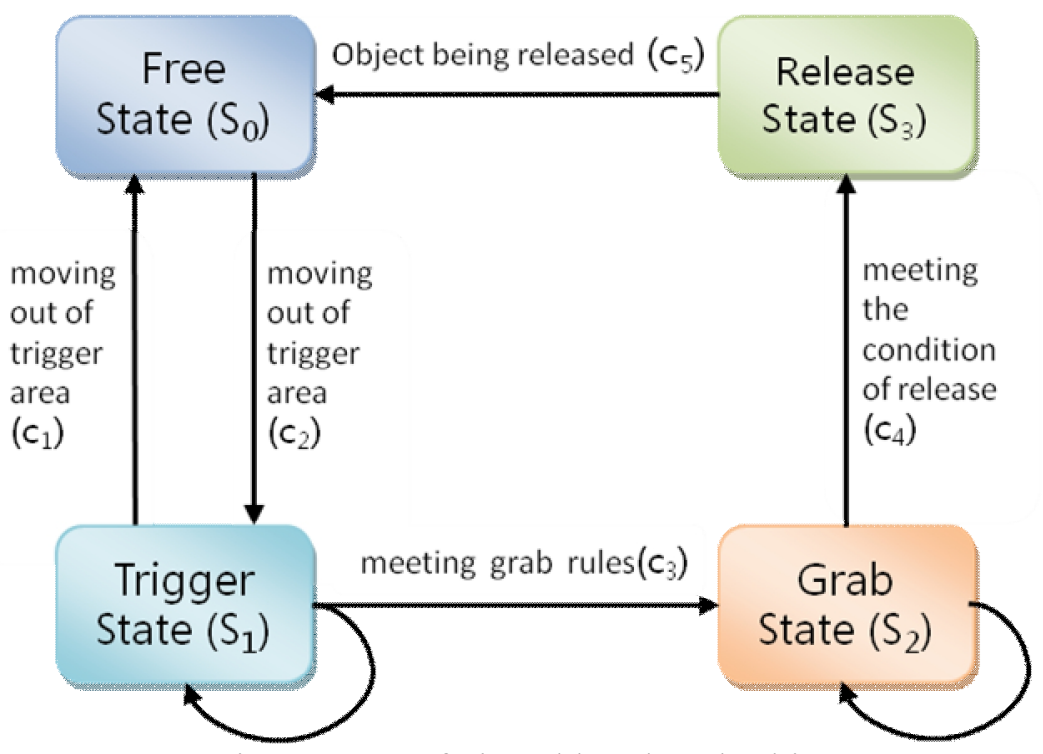

Fig. 1 FSM of virtual hand grab objects

According to the principle of FSM, the constituent elements of FSM are defined as: states (S), input events (X), output(Y), states transition function (f) and output function (g).

States (S): grab states of virtual hand. There are the free state, trigger state, grab state and release state. Each state corresponds to a kind of grab state between the virtual hand and the grabbed object.

Input events (X): correspond to the five states transition conditions shown in Fig. 1. There are the conditions of the virtual hand moving into the object grab trigger area, the virtual hand moving out of the object grab trigger area, the virtual hand meeting the rules of grab, the virtual hand meeting the condition of release and the object being released.

Output(Y): the rendering results displayed to the operator.

States transition function (f): defined as the determination function which determines the states transition from the current state to the next one. Eq. 1 shows its relationship with the states and time, where $X(t) \in X, S(t) \in S$.

$$
S(t+1)=f(X(t), S(t))
$$

Output function (g): defined as a mapping relationship between the current state and the output. Eq. 2 shows its relationship with the states, where $Y(t) \in Y$.

$Y(t)=g(X(t), S(t))$

The description of each state is as follows:

\section{The Free State $\left(\mathbf{S}_{\mathbf{0}}\right)$ :}

In the free state, the virtual hand does not grab any object. In this condition, virtual hand can move freely and the finger joints can bend freely, as is shown in Fig. 2. 
The Trigger State $\left(S_{1}\right)$ :

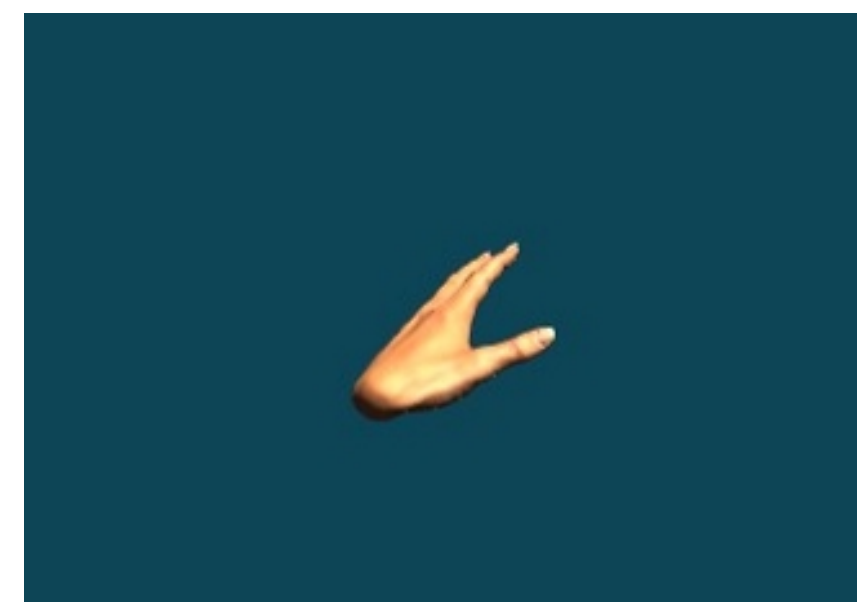

Fig. 2 Virtual hand movement under the free state

In the trigger state, the virtual hand moves into the object grab trigger area, but does not meet the grab determining rules. At this time, the virtual hand does not touch the object, so that the object to be grabbed does not move, as is shown in Fig. 3.

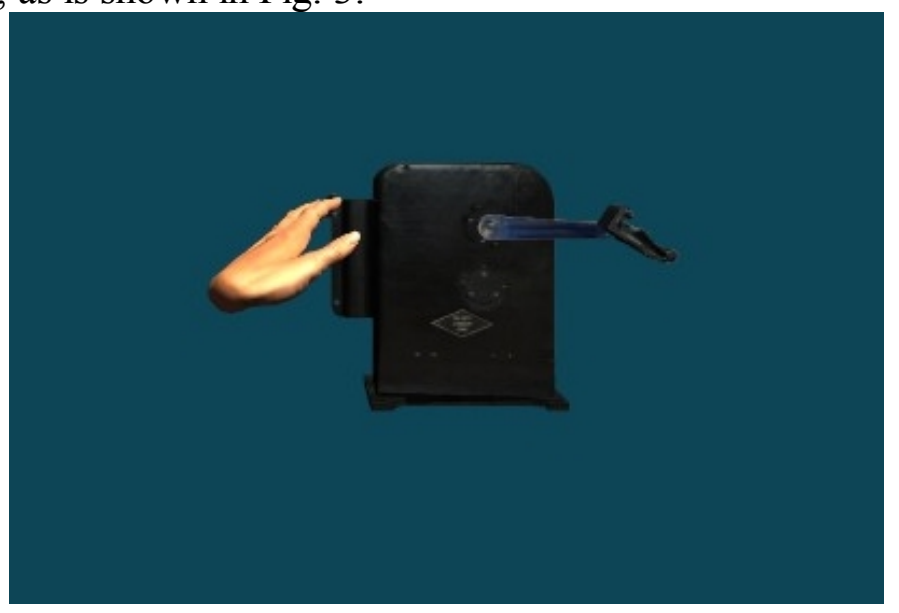

Fig. 3 The virtual hand and object in the trigger state

\section{The Grab State $\left(\mathbf{S}_{2}\right)$ :}

In the grab state, the virtual hand can grab object stably by following the two grab determining rules. The grabbed object is adsorbed to virtual hand in the grab state, moving and rotating with the virtual hand together as a child node, as is shown in Fig. 4.

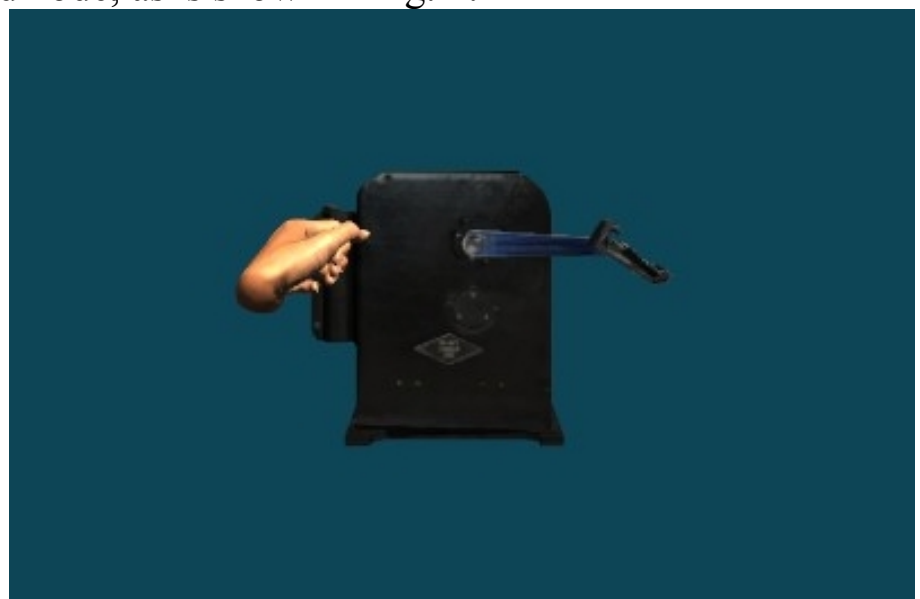

Fig. 4 The virtual hand and grabbed object in the grab state 


\section{The Release State $\left(\mathbf{S}_{3}\right)$ :}

The virtual hand converts to the release state when it does not satisfy Rule Two of the grab determining rules after grabbing the object. In this state, the virtual hand releases the grabbed object and then converts to the free state, as is shown in Fig. 5.

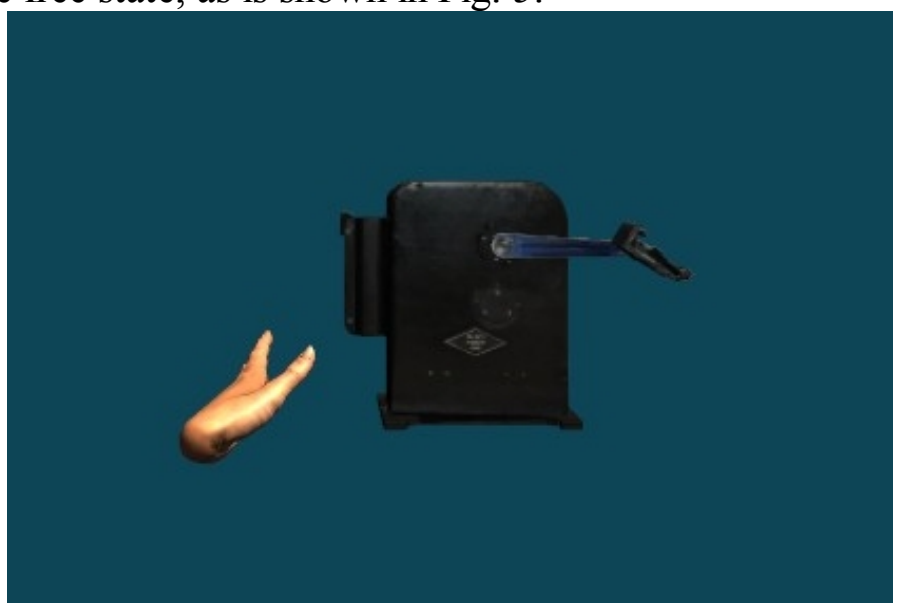

Fig. 5 The virtual hand and released object in the release state

A typical grab process is shown in Fig. 6. The virtual hand is in the free state at the beginning, then moves into the object grab trigger area and converts to the trigger state. The virtual hand can grab the object to convert to the grab state by following the two grab determining rules. When the virtual hand does not satisfy Rule Two, it releases the object and converts to the release state.

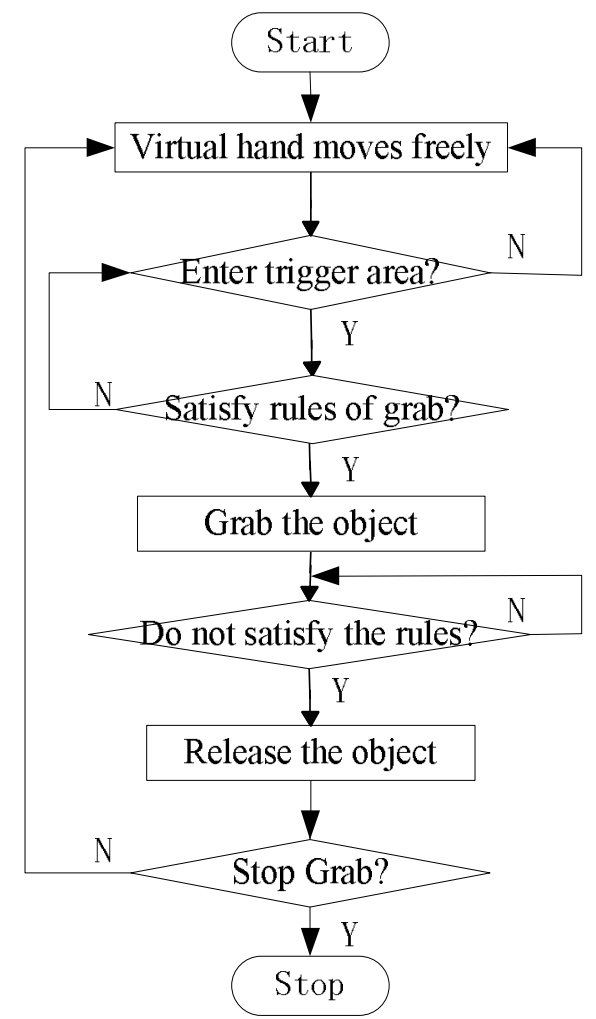

Fig. 6 The process of virtual hand grabbing

\section{Experiment and Result}

In order to test our grab rules and grab simulation FSM model, we analyzed 358 times grabbing operation from 40 subjects. We took the average number of grabbing operation every successful grab and the average grabbing time as the evaluation. The result is shown in Table 1. 
Table 1 Evaluation of virtual hand grab

\begin{tabular}{|c|c|c|}
\hline Evaluation & Average & Standard deviation \\
\hline Numbers of grabbing operation every successful grab & 1.50 & 0.984 \\
\hline Grabbing time (seconds) & 4.41 & 3.364 \\
\hline
\end{tabular}

From the evaluation above we can concluded that the virtual hand grabbing operation, which following our grab determining rules and FSM model, has a high success rate and efficiency. This method can be used as astronaut grabbing operation training method in a virtual weightless environment.

\section{Summary}

In this paper, grab determining rules and a FSM model were proposed to simulate grabbing operation in a weightless virtual environment. This method suited virtual weightless training well and can be widely used in astronaut virtual training. However, the success rate and efficiency of grabbing operation is also concerned about the shape of the grabbed object [8]. In the future work, we will also take object's shape into consideration to improve the interactivity of astronaut virtual training system.

\section{References}

[1] A. K. Noor. Potential of virtual worlds for remote space exploration [J]. Advances in Engineering Software, 2010, 41: 666-673.

[2] H. Aoki, C. Oman, A. Natapoff. Virtual-reality-based 3D navigation training for emergency egress from spacecraft [J]. Aviation, Space and Environmental Medicine, 2007, 78(8):774-783.

[3] H. Hu, J. Chao, Z. Zhao.Study of Vision-Based Hand Gesture Recognition System for Astronaut Virtual Training[J]. Advanced Materials Research. 2014, 998: 1062-1065.

[4] Y. Liu, S. Chen, G. Jiang, et al. VR Simulation System for EVA Astronaut Training [C]. //Proceedings of AIAA SPACE 2010 Conference \& Exposition, 2010.

[5] I. A. Twombly, J. Smith, et al. NASA Virtual Glovebox: An Immersive Virtual Desktop Environment for Training Astronauts in Life Science[C]. // Proceedings of 7th Multi-Conference on Systemics, Cybernetics and Informatics, 2003.

[6] A. T. Bouloutas, G. W. Hart, M. Schwartz. Fault identification using a finite state machine model with unreliablepartially observed data sequences [J]. IEEE Transactions on Comm unications, 1993, 41(7): 1074-1083.

[7] Y. Chen, F. Lin. Safety control of discrete event systems using finite state machines with parameters [C]. //P roceedings of the American Control Conference. Arlington: American Auotmatic Control Council, 2001: 975-980

[8] Z. Feng, B. Yan, T. Xu, et al. 3D direct human-computer interface paradigm based on free hand tracking [J]. Chinese Journal of Computers, 2014, 37(6): 1309-1323. 